ARTÍCULOS ORIGINALES

Rev Chil Salud Pública 2014; Vol 18 (2): 173-182

\section{MORTALIDAD POR INFLUENZA pA(H1N1) 2009 EN EL NORESTE DE MÉXICO}

\author{
Mortality from PaNDEMIC INFLUENZA A (H1N1) IN NORTH \\ EAST OF MeXico
}

\section{RESUMEN}

Objetivo. Determinar los factores asociados a la mortalidad por influenza $p A(H 1 N 1)$ en los pacientes hospitalizados por infección respiratoria aguda grave (IRAG) confirmada por reacción en cadena de la polimerasa (PCR) en el Instituto Mexicano del Seguro Social (IMSS).

Material y métodos. En el IMSS en la delegación de Nuevo León entre el 1 de junio de 2009 y 9 de marzo de 2010 se realizó un estudio observacional retrospectivo de casos y controles, utilizando la base de datos del Sistema de Información en Linea para la Vigilancia Epidemiológica de Influenza (SINOLAVE). Se incluyeron 278 pacientes hospitalizados con IRAG (controles) y 50 pacientes con IRAG que fallecieron (casos) debido a la infección por virus influenza $p A(H 1 N 1)$.

Resultados. Los factores asociados a la mortalidad en los pacientes hospitalizados por IRAG debida a influenza $p A(H 1 N 1)$ fueron la edad (OR: 1,03 IC95\% 1,01-1,05) y la obesidad (OR: 4,44 IC95\% 1,85-1,6), utilizando un modelo de regresión logística.

Conclusión. Podemos concluir que en la delegación de Nuevo León del IMSS, la influenza $p A(H 1 N 1)$ afectó principalmente a adultos jóvenes, sin embargo las muertes se presentaron en mayor número en los pacientes al incrementar la edad y en pacientes con alguna comorbilidad.

Palabras clave: Influenza pandémica A(H1N1), mortalidad, infección respiratoria aguda grave, factores de riesgo, razón de probabilidad.

\section{ABSTRACT}

Objective. To determine factors associated with mortality from $p A$ influenz $A(H 1 N 1)$ - confirmed by polymerase chain reaction (PCR) - In hospitalized patients with severe acute respiratory infection (SARI) in the Mexican Social Security Institute (IMSS). 
Methods. In the IMSS in the Delegation of Nuevo Leon between June 1, 2009 and March 9, 2010 a retrospective observational case-control study was conducted using the database of Online Information System for Epidemiological Surveillance of Influenza (SINOLAVE). 278 inpatients with SARI (controls) and 50 SARI patients who died (cases) due to infection with influenza virus $p A(H 1 N 1)$ were included.

Results. In the logistic regression model factors associated with mortality in patients hospitalized due to SARI pA influenzA(H1N1) were age (OR: 1.03 95\% CI 1.01-1.05) and obesity (OR: 4.44951 .85 to $1 \%, 0.6)$.

Conclusion. We can conclude that the delegation of Nuevo León of the IMSS, pA influenz $A(H 1 N 1)$ affects mainly young adults, though the deaths occurred in greater numbers in patients with increasing age and in patients with comorbidities.

Key words: pandemic influenza $\mathrm{A}(\mathrm{H} 1 \mathrm{~N} 1)[\mathrm{pA}(\mathrm{H} 1 \mathrm{~N} 1)]$, mortality, severe acute respiratory infection, risk factors, Odds ratio.

\section{INTRODUCCIÓN}

La influenza es una enfermedad respiratoria aguda producida por un virus de ácido ribonucleico (ARN), de la familia de los Orthomyxoviridae. Se clasifica en tres tipos: la influenza $\mathrm{A}, \mathrm{B}$ y $\mathrm{C}$. El virus influenza tipo A, muta más rápidamente y por lo tanto muestra mayor flexibilidad antigénica y mayor virulencia que las variedades B y C. El virus influenza A se subclasifica en subtipos por su hemaglutinina $(\mathrm{H})$ y neuroaminidasa $(\mathrm{N})$. El virus de la influenza A es el principal causante de las epidemias invernales que se repiten cada año y de pandemias cada 10 o 20 años, debidas a cambios antigénicos en su envoltura y que han ocasionado elevadas tasas de mortalidad en los seres humanos. ${ }^{1}$ El período de incubación de la gripe es en promedio de tres días. Las manifestaciones clínicas típicas de la influenza son fiebre elevada, cefalea, tos, mialgias, artralgias, odinofagia y rinitis. Se transmite con rapidez durante la temporada invernal y afecta del 10 al $20 \%^{2}$ de la población y hasta el $50 \%$ de la población en una pandemia, ocasionando mayor número de muertes. ${ }^{3}$

La morbilidad y mortalidad por influenza estacional es bien comprendida y se han identificado los grupos vulnerables, como son los individuos mayores de 65 años y niños menores de dos años de edad principalmente. ${ }^{4,5}$ Otros factores de riesgo que se han asociado con la IRAG debida a influenza pandémica $\mathrm{A}(\mathrm{H} 1 \mathrm{~N} 1)$ [pA(H1N1)] durante el 2009 fueron: la obesidad mórbida (con índice de masa corporal $\geq 40$ ), las enfermedades pulmonares (como asma y la enfermedad pulmonar obstructiva crónica), las enfermedades cardiovasculares (las enfermedades cardiacas congénitas, excepto la hipertensión arterial), las enfermedades metabólicas (diabetes mellitus), las enfermedades renales, las enfermedades hepáticas, las enfermedades hematológicas (la enfermedad de células falciformes), las enfermedades neurológicas (la parálisis cerebral, la epilepsia, los accidentes cerebrovasculares, la discapacidad intelectual, el retraso en el desarrollo de moderado a grave y la distrofia muscular), las inmunodeficiencias (cáncer, el virus de inmunodeficiencia humana, etcétera), los indios americanos y los nativos de Alaska, ${ }^{6,7}$ que se vinculan a menudo con un aumento en las tasas de hospitalización y de mortalidad. ${ }^{8,9}$

Actualmente se ha documentado que el embarazo es un factor de riesgo para desarrollar formas graves de la enfermedad, y se ha visto que el riesgo de morir por influenza se incrementa durante el tercer trimestre del embarazo. ${ }^{10,11}$ La vacuna de influenza disminuye la mortalidad por una infección respiratoria aguda grave (IRAG) debida a influenza en población de alto riesgo de presentar complicaciones, y el tratamiento acorta la duración 
de la enfermedad y mejora la condición clínica del paciente, evitando complicaciones.

El estudio incluyó las comorbilidades identificadas durante la pandemia de influenza AH1N1 en 2009, utilizando el total de pacientes con una IRAG ocurrida por influenza pA(H1N1) entre el 1 de junio del 2009 y el 9 de marzo del 2010, con el objetivo de identificar en la delegación de Nuevo León a la población de mayor riesgo de morir por una IRAG debida a influenza pA(H1N1). No se han hecho publicaciones similares con esta población. Se pretende mejorar el proceso de atención médica que se otorga al $60 \%$ de los habitantes del estado y evitar desenlaces fatales que afecten a las familias derechohabientes.

\section{MATERIAL Y MÉTODOS}

\section{Diseño}

Estudio observacional retrospectivo de casos y controles. Se realizó un censo y se incluyeron todos los pacientes hospitalizados por la IRAG por influenza $\mathrm{pA}(\mathrm{H} 1 \mathrm{~N} 1)$ confirmada por técnica de biología molecular en muestras respiratorias (reacción en cadena de la polimerasa o PCR) en la delegación de Nuevo León del IMSS.

\section{Población}

Se incluyeron en el presente estudio todas las IRAG hospitalizadas debidas al virus de influenza $\mathrm{pA}(\mathrm{H} 1 \mathrm{~N} 1)$, notificadas a través de la plataforma Sistema de Información en Línea para la Vigilancia Epidemiológica de Influenza (SINOLAVE) entre el 1 de junio 2009 y el 9 de marzo de 2010.

Criterios de inclusión. Pacientes hospitalizados, de cualquier edad, con la IRAG debi$\mathrm{da}$ al virus influenza $\mathrm{pA}(\mathrm{H} 1 \mathrm{~N} 1)$ confirmada por técnica de PCR de muestras respiratorias en el IMSS en la delegación de Nuevo León, que sea derechohabiente del IMSS.

Criterios de exclusión. Todos los casos sospechosos, los casos confirmados de influenza estacional A (H3N2), los casos no ocasionados por influenza pA(H1N1), los casos confirmados y tratados ambulatoriamente, los casos con enfermedad tipo influenza (persona de cualquier edad que presente o refiera haber tenido fiebre mayor o igual a $38^{\circ} \mathrm{C}$, tos y cefalea, acompañadas de uno o más de los siguientes signos o síntomas: rinorrea, coriza, artralgias, mialgias, postración, odinofagia, dolor torácico, dolor abdominal, congestión nasal o diarrea. En menores de cinco años de edad, se considera como un signo cardinal la irritabilidad, en sustitución de la cefalea. En mayores de 65 años, no se requerirá la fiebre como síntoma cardinal).

Definición operacional de control. Fue todo paciente hospitalizado con una IRAG debida al virus de influenza pA(H1N1), confirmada por técnica de biología molecular en muestras respiratorias (reacción en cadena de la polimerasa o PCR). La IRAG se define como toda persona de cualquier edad que presenta dificultad al respirar con antecedente de fiebre $\geq 38^{\circ} \mathrm{C}$, con uno o más de los siguientes síntomas: ataque al estado general, dolor torácico o polipnea.

Definición operacional de caso. Se consideró todo caso con una IRAG que falleció con un resultado positivo a la prueba confirmatoria de influenza pA.(H1N1).

Definición comorbilidades. Se interrogó directa o indirectamente a cada uno de los pacientes sobre la presencia o ausencia de enfermedad concomitante.

Epo. Es un trastorno pulmonar que se caracteriza por la existencia de una obstrucción de las vías aéreas en forma progresiva y no reversible. Está constituida por la bronquitis crónica y enfisema.

Asma. El asma es una enfermedad crónica que se caracteriza por ataques recurrentes de disnea y sibilancias, que varían en severidad y frecuencia de una persona a otra. Los síntomas pueden sobrevenir 
varias veces al día o a la semana, y en algunas personas se agravan durante la actividad física o por la noche.

Inmunodeficiencia. Cualquier trastorno en la fisiología de la inmunidad celular o humoral. Las inmunodeficiencias pueden ser congénitas, que generalmente obedecen a trastornos en el desarrollo de las células inmunitarias, a carencia o ausencia de inmunoglobulinas o a alteraciones de sistemas efectores como el complemento o la fagocitosis. Las inmunodeficiencias adquiridas, por su parte, son secundarias a infecciones víricas (virus de inmunodeficiencia humana, VIH), enfermedades subyacentes como leucemias o linfomas, tratamiento con fármacos inmunosupresores, malnutrición.

Obesidad. De acuerdo a la OMS, la obesidad se define por la presencia de un Índice de Masa Corporal igual o superior a $30 \mathrm{~kg} / \mathrm{m}^{2}$.

Diabetes. Es un trastorno metabólico que tiene causas diversas. Se caracteriza por hiperglucemia crónica y trastornos del metabolismo de los carbohidratos, las grasas y las proteínas como consecuencia de anomalías de la secreción o del efecto de la insulina.

\section{Recolección de datos}

Para facilitar la notificación de pacientes con una probable IRAG debida a influenza pA(H1N1) a la Dirección General de Epidemiología (DGE), el IMSS implementó el "estudio epidemiológico de casos sospechosos de influenza" en una plataforma electrónica llamada Sistema de Información en Línea para la Vigilancia Epidemiológica de Influenza (SINOLAVE). Cada encargado de la vigilancia epidemiológica en su unidad médico-hospitalaria recababa la información clínico-epidemiológica del caso clínico con IRAG, tomaba la muestra para la confirmación de la enfermedad y registraba el caso en la plataforma.
El estudio contenía información sobre las características epidemiológicas (edad, género, lugar de procedencia, ocupación, fecha de inicio de los síntomas), clínicas (signos y síntomas), comorbilidades y/ factores de riesgo como: diabetes, enfermedad pulmonar obstructiva crónica (EPOC), asma, inmunosupresión y de otras variables como: embarazo tratamiento con inhibidores neuroaminidasa y el antecedente vacunal con vacuna antiinfluenza.

\section{Laboratorio}

Un resultado positivo a la influenza A(H1N1) 2009 mediante la prueba de Reacción en Cadena de la Polimerasa en Transcriptasa Reversa (rRT-PCR) en tiempo real, fue considerado como prueba confirmatoria. Las muestras para confirmación de la infección por influenza $\mathrm{pA}(\mathrm{H} 1 \mathrm{~N} 1)$ se obtuvieron mediante hisopado nasofaríngeo o se tomaron muestras del tracto respiratorio inferior (broncoscopía, lavado bronquio-alveolar, etcétera). Cuando fue posible, se tomaron muestras post mortem por hisopado nasofaríngeo o muestras de tejido pulmonar durante la necropsia.

\section{Aspectos éticos}

El estudio epidemiológico de "Casos Sospechosos de Influenza” es el formato normado por la Secretaría de Salud para realizar la notificación de casos; esta investigación formó parte del Plan de Contingencia Institucional en Respuesta a la Epidemia de Influenza Pandémica A(H1N1) 2009, por lo que no se consideró necesaria una evaluación ética específica ni solicitar consentimiento informado; cada registro fue foliado, eliminando el nombre y apellidos de los pacientes para su análisis.

\section{ANÁLISIS DE DATOS}

Con la base de datos creada de todos los casos confirmados con influenza pA(H1N1) en el SINOLAVE se realizó la descripción 
de las variables cuantitativas (edad, tiempo de atención médica), utilizando la media o la mediana, y para la descripción de variables cualitativas (género, ocupación, lugar de residencia) se describieron a través de porcentajes. En el análisis bivariado, las variables cuantitativas discretas como la edad y el tiempo de atención médica no mostraron una distribución normal en uno de los grupos (con la prueba de Shapiro Wilks), por lo que se compararon ambos grupos utilizando la prueba no paramétrica de Mann-Whitney. Las variables cualitativas nominales (EPOC, asma, diabetes, inmunodeficiencia, obesidad, embarazo, antecedente vacunal de vacuna anti-influenza y tratamiento con inhibidores neuroaminidasa) se dicotomizaron (1=presente, $0=$ ausente) y se compararon entre ambos grupos, utilizando la prueba exacta de Fisher, para evaluar su asociación. Se creó un modelo con las variables que mostraron una diferencia estadísticamente significativa (diabetes, obesidad, tiempo de atención médica). Además se incluyeron otras variables como el asma y la inmunodeficiencia, porque existen fundamentos científicos para identificar estas variables como factores de riesgo de morir por influenza $\mathrm{pA}(\mathrm{H} 1 \mathrm{~N} 1){ }^{8,12,13}$ y se incluyeron dentro del modelo final. Se excluyó el embarazo y la EPOC porque no se notificaron defunciones con estas comorbilidades en nuestra población durante el estudio. Finalmente el modelo de regresión logística múltiple incluyó la variable edad, el tiempo de atención médica, diabetes, obesidad, inmunodeficiencia y asma. Este modelo se evaluó a través de las pruebas estadísticas de la razón de verosimilitud (Likelibood ratio test) y la prueba de $\mathrm{Xi}^{2}$ de Hosmer y Lemeshow. El análisis estadístico se realizó con el paquete estadístico STATA11.

\section{RESULTADOS}

Durante el estudio se confirmaron un total de 1.236 casos de influenza pA(H1N1), el $77,5 \%(958 / 1236)$ de los pacientes fue tratado ambulatoriamente y 22,5\% (278/1236) hospitalizado. De los pacientes hospitaliza- dos el 18\% (50/278) falleció en el IMSS de la delegación de Nuevo León. Entre los pacientes que fallecieron por influenza $\mathrm{pA}(\mathrm{H} 1 \mathrm{~N} 1)$, la mediana de edad fue de 47 años en comparación con la mediana de 40 años de los sujetos controles $(p=0001)$. El tiempo transcurrido entre el inicio de los síntomas y su primer contacto con los servicios de salud fue de cuatro días en los sujetos casos en comparación con los tres días de los pacientes hospitalizados por IRAG debida a influenza $\mathrm{pA}(\mathrm{H} 1 \mathrm{~N} 1)(\mathrm{p}=0.03)$. La mayor proporción de los sujetos de ambos grupos vive en el área Metropolitana de Monterrey. Al comparar las comorbilidades en ambos grupos se encontró una prevalencia mayor de diabetes en los pacientes que fallecieron $22 \%(11 / 50)$ ) en comparación con los controles cuya prevalencia fue del 11\% (26/233) ( $\mathrm{p}=0,04)$. También se encontró una mayor prevalencia de obesidad en las defunciones por IRAG debida a influenza pA(H1N1) (el 26\% (13/50) $(\mathrm{p}=0,001))$. No se notificó ninguna defunción materna por una IRAG debida a influenza pA(H1N1). De un total de 15 mujeres en edad fértil que estaban embarazadas, 13 (87\%) estaban cursando el tercer trimestre de embarazo y $2(13 \%)$ el segundo trimestre. No se observó una diferencia estadísticamente significativa entre ambos grupos en las siguientes variables clínico-epidemiológicas: el embarazo $(p=0,06)$, el antecedente de inmunización con vacuna antiinfluenza de virus muertos $(p=1,00)$, el uso de terapia antiviral con inhibidores de neuroaminidasa $(\mathrm{p}=0,92)$, el antecedente de EPOC $(\mathrm{p}=0,61)$, el asma $(p=0,69)$ y la inmunodeficiencia $(p=1,00)$ entre ambos grupos (Cuadro 1). Al obtener la razón de probabilidad cruda, los pacientes hospitalizados con IRAG debida a influenza $\mathrm{pA}(\mathrm{H} 1 \mathrm{~N} 1)$ diabéticos tienen 2,24 veces más posibilidades de morir por influenza pA(H1N1) (IC95\% 1,02-4,95). Los pacientes controles con antecedente de obesidad tuvieron 4,19 veces (IC95\% 1,85-9,49) más posibilidades de morir por influenza pA(H1N1), en comparación con los que tenían peso normal o sobrepeso. En esta población, los factores de riesgo como asma (OR 0,46 IC 95\% 0,05-3,65), inmunodeficiencia 
(OR 0,92 IC 95\% 0,26-3,34) y EPOC no se asociaron a mayor riesgo de morir por influenza pA(H1N1) (Cuadro 2).

\section{Análisis multivariado}

En los sujetos controles, por cada año que incrementan su edad tienen 1,03 veces más posibilidades de morir por IRAG debido a influenza $\mathrm{pA}(\mathrm{H} 1 \mathrm{~N} 1)$ (IC 95\% 1,01- 1,05), ajustado el modelo por atención médica, asma, diabetes, obesidad e inmunodeficiencia. Los pacientes controles que tenían el antecedente de obesidad tenían 4,5 (IC 95\% 1,8610,6) veces más posibilidades de morir por influenza $\mathrm{pA}(\mathrm{H} 1 \mathrm{~N} 1)$ en comparación con los sujetos que tenían sobrepeso o peso normal, ajustando el modelo a la edad, tiempo de atención médica, diabetes, asma e inmunodeficiencia. Los sujetos controles hospitalizados con IRAG debido a influenza pA(H1N1), por cada día que pasa desde el inicio de sus síntomas hasta su hospitalización, tienen 1,03 veces más posibilidades de morir debido a influenza pA(H1N1) (IC 95\% 1,00-1,07), ajustado el modelo por edad, diabetes asma, obesidad e inmunodeficiencia. Una vez ajustado el modelo por las variables edad, tiempo de atención médica, asma e inmunodeficiencia, la diabetes ${ }^{24}$ (OR 1,17 IC 95\% 0,47-2,94), asma (OR 0,61 IC 95\% 0,73-5,18) e inmunodeficiencia (OR 0,98 IC 95\% 0,25-3,76), no incrementan el riesgo de morir por influenza pA(H1N1) en esta población (Cuadro 3).

\section{DISCUSIÓN}

Los resultados del presente estudio indican que la mortalidad por influenza pA(H1N1) está asociada directamente a la edad del paciente y al antecedente de obesidad.

La mediana de edad de los pacientes que fallecieron por una IRAG debida a influenza pA(H1N1) fue de 47 años, y la mediana de edad fue de 30 años en los pacientes hospitalizados con IRAG por influenza pA(H1N1). Lo anterior se relaciona con lo que concluyó F. Dolci y cols. "El nuevo virus A(H1N1) produce mayor mortalidad en personas jó- venes", al contrario de lo que sucede con la influenza estacional que muestra un mayor impacto en niños pequeños y personas de edad avanzada". ${ }^{2}$ Este estudio demuestra que los pacientes hospitalizados por IRAG debida a influenza pA(H1N1) tienen más posibilidades de morir por influenza $\mathrm{pA}$ H1N1 conforme incrementa la edad. Esto puede deberse a que con la edad se incrementa la probabilidad de presentar una o más comorbilidades e incrementa su prevalencia con la edad. ${ }^{13}$

Existió una mayor prevalencia de obesidad en los fallecidos por IRAG debida a influenza pA(H1N1) (26\%) en comparación con los pacientes hospitalizados (8\%). Podemos afirmar que la obesidad es un factor de riesgo de morir por influenza pA(H1N1) (OR 3,53 IC 95\% 1,51-7,79) en la población estudiada. Morgan OW y cols. "Advierten que el riesgo de morir luego de la infección por virus influenza $\mathrm{A}(\mathrm{H} 1 \mathrm{~N} 1)$ puede ser mayor para las personas con obesidad mórbida”. ${ }^{14,15,16}$ Actualmente la obesidad es un problema de Salud Pública en México, más del $70 \%$ de la población tiene sobrepeso u obesidad; en el estado de Nuevo León la obesidad y sobrepeso tienen una prevalencia semejante. ${ }^{17}$ Una limitante de nuestro estudio fue que no se categorizó la variable obesidad por la falta del índice de masa corporal, por lo que pudiéramos sobreestimar el riesgo de morir por influenza $\mathrm{A}(\mathrm{H} 1 \mathrm{~N} 1)$ en los pacientes con obesidad.

Otros factores de riesgo que se han relacionado a la mortalidad por influenza pA(H1N1) son las enfermedades cardiovasculares y respiratorias crónicas, incluyendo asma y EPOC, y las inmunodeficiencias, pero en este estudio se encontró baja o nula frecuencia de fallecimientos por IRAG debi$\mathrm{da}$ a influenza $\mathrm{pA}(\mathrm{H} 1 \mathrm{~N} 1)$ asociados a estás comorbilidades. Esto se puede explicar por errores de clasificación de los factores de riesgo en el estudio epidemiológico, por estudios epidemiológicos incompletos, por la mediana de edad, que fue 47 años en las defunciones confirmadas por influenza $\mathrm{pA}(\mathrm{H} 1 \mathrm{~N} 1)$, la mayoría adultos jóvenes, sin antecedentes mórbidos de importancia. 
El embarazo no constituyó un factor de riesgo de morir en las mujeres en edad fértil que se hospitalizaron con IRAG debida a influenza $\mathrm{pA}(\mathrm{H} 1 \mathrm{~N} 1)$ durante el período estudiado. Van Kerkhove M. y cols. señalaron que el mayor riesgo de complicaciones se presenta al final del embarazo. "Las mujeres embarazadas en cualquier trimestre tienen 1,9 posibilidades de morir por influenza A(H1N1)". El riesgo de morir por influenza en el embarazo se incrementa con el transcurso del embarazo. En el primer trimestre la posibilidad es 0,9 , en el segundo 2,5 y en el tercer trimestre 16,5. En nuestro estudio se encontró que el $24 \%$ de las mujeres en edad fértil hospitalizadas por influenza pA(H1N1) estaba embarazada, el $80 \%$ tenía 32 años o menos, y el $87 \%$ cursaba el tercer trimestre del embarazo, lo que concuerda con la literatura. En este contexto clínico, el IMSS reportó una letalidad del 6,3\% durante el embarazo. ${ }^{18,19}$

La vacunación contra la influenza estacional no parece ser un factor protector. El antecedente vacunal de la influenza estacional no ha demostrado aumentar o disminuir el riesgo de contraer la influenza pandémica. ${ }^{20}$ Se recomendó iniciar el tratamiento antiviral durante la pandemia dentro de las primeras 48 horas de iniciados los síntomas para reducir el riesgo de complicaciones (neumonias, insuficiencia respiratoria o muerte). ${ }^{21}$

El estudio cuenta con validez interna, los casos y controles se originan de la misma fuente: de los hospitales del IMSS, lo que permite controlar algunas fuentes de sesgo o errores de selección. Consideramos que estos resultados no pueden ser extrapolados a la población en general, porque algunos estudios epidemiológicos estaban incompletos, generando celdas vacías en la base de datos que pudieran haber afectado el análisis de la información. Además, se empleó un formato ya estandarizado, el cual no permitió evaluar ciertas variables como la obesidad en forma exhaustiva. Otras fuentes de sesgo pudieron haberse presentado por realizar interrogatorios indirectos a familiares cuando las condiciones físicas del paciente no lo permitieron y al llenar el estudio epidemiológico cometer errores de calificación en las comorbilidades.

\section{CONCLUSIÓN}

Podemos concluir que en la delegación de Nuevo León del IMSS, la influenza pA(H1N1) afectó principalmente a adultos jóvenes; sin embargo las muertes se presentaron en mayor número en los pacientes al incrementar la edad y en personas con algún factor de riesgo. En el período del estudio no se presentaron muertes maternas por influenza pA(H1N1), lo que generaría una nueva fuente de investigación.

Este análisis espera ser de utilidad para los profesionales de la salud a cargo de tomar decisiones, brindándoles información de los grupos vulnerables donde se deben fortalecer las acciones de prevención y control de la enfermedad por influenza $\mathrm{pA}(\mathrm{H} 1 \mathrm{~N} 1)$.

\section{REFERENCIAS}

1. Barrera A, Cortés V, Castaño R, Díaz R, Martínez C, Medécigo A, et al. Prevención, diagnóstico y tratamiento de influenza A(H1N1). Guía de práctica clínica. México: Instituto Mexicano del Seguro Social; 2009. pp. 1-56.

2. Cabello A, Von Horoch M, Ojeda A, Troche G, Morel G, Samudio M. et al. Factores asociados a mortalidad en la pandemia de influenza H1N1 2009. Mem Inst Investig Cienc Salud. 2011; 7: 5-12.

3. Sociedad Argentina de Infectología. Sociedad Argentina de Pediatría. Documento sobre infección por virus de influenza A(H1N1). Argentina: SADI, SAP; 2009. pp. 13-60.

4. Acuña G. Historia y amenazas. Rev Chil Infect. 2004; 21: 162-4.

5. Thompson WW, Shay DK, Weintraub E, Brammer L, Cox N, Anderson LJ, et al. Mortality associated with influenza and respiratory syncytial virus in the United States. JAMA. 2003; 289(2): 179-86.

6. National Center for Immunization and Respiratory Diseases. Use of influenza A(H1N1) 2009 monovalent vaccine. Recommendations of the Advisory Committee on Immunization Practices (ACIP), 2009. MMWR Recomm Rep. 2009; 58: 1-8. 
7. Mereckiene J, Cotter S, Nicoll A, LevyBruhl D, Ferro A, Tridente G, et al. National seasonal influenza vaccination survey in Europe, 2008. Euro Surveill. 2008; 13: 19017.

8. Kusznierz GF, Imaz MS, Zerbini EV, Savy V, Knez V, Sequeira MD. Effect of influenza epidemics on mortality in Santa Fe, Argentina, during 1992-1999. Rev Panam Salud Pública. 2002; 12: 26-36.

9. Hollenbek JE. An avian connection as a catalyst to the 1918-1919 influenza pandemic. Int J Med Sci. 2005; 2: 87-90.

10. Dodds L, McNeil SA, Fell DB, Allen VM, Coombs A, Scott J, et al. Impact of influenza exposure on rates of hospital admissions and physician visits because of respiratory illness among pregnant women. CMAJ. 2007; 176: 463-8.

11. Harris J. Influenza occurring in pregnant women. JAMA. 1919; 72: 978-80.

12. Fajardo-Dolci G, Hernández F, Santacruz J, Lamy P, Arboleya H, Gutiérrez R, et al. Perfil epidemiológico de la mortalidad por influenza humana A(H1N1) en México. Salud Pública Mex. 2009; 51: 361-71.

13. Santa-Olalla Peralta P, Cortés García M, Limia Sánchez A, Andrés Prado J, Pachón del Amo I, Sierra Moros MJ. Casos de infección por gripe pandémicA(H1N1) 2009. Hospitalizados en cuidados intensivos en España: factores asociados a riesgo de muerte, abril 2009-enero 2010. Rev Esp Salud Pública. 2010; 84(5): 547-567

14. Piñón-Ramos A, Oropesa-Fernández $S$, Aragonés-López C, Galindo B, AcostaHerrera B, Hernández-Espinosa B. Influenza y vacunación. Rev Biomed. 2005; 16: 45-53.
15. De Haro López C, Ferrer Roca R, Vallés Daunis J. Neumonía y síndrome de distres respiratorio agudo producido por el virus influenza $\mathrm{A}(\mathrm{H} 1 \mathrm{~N} 1)$. Med Intensiva. 2009; 33: 455-8.

16. Morgan OW, Bramley A, Fowlkes A, Freedman DS, Taylor TH, Gargiullo P, et al. Morbid obesity as a risk factor for hospitalization and death due to 2009 pandemic influenza A(H1N1) disease. PLoS One. 2010; 5(3): e9694.

17. Louie JK, Acosta M, Winter K, Jean C, Gavali $\mathrm{S}$, Schechter $\mathrm{R}$, el al. Factors associated with death or hospitalization due to pandemic 2009 influenza $\mathrm{A}(\mathrm{H} 1 \mathrm{~N} 1)$ infection in California. JAMA. 2009; 302: 1896-902.

18. Echevarría-Zuno S, Mejía-Aranguré JM, Mar-Obeso AJ, Grajales-Muñiz C, RoblesPérez E, González-León M, et al. Infection and death from influenza A H1N1 virus in Mexico: a retrospective analysis. Lancet. 2009; 374: 2072-9.

19. Van Kerkhove MD, Vandemaele KA, Shinde V, Jaramillo-Gutierrez G, Koukounari A, Donnelly CA, et al. Risk factors for severe outcomes following 2009 influenza A(H1N1) infection: A global pooled analysis. PLoS Med. 2011; 8(7): e1001053.

20. Centers for Disease Control and Prevention (U.S.). Effectiveness of 2008-09 trivalent influenza vaccine against 2009 pandemic influenza $\mathrm{A}(\mathrm{H} 1 \mathrm{~N} 1)$ - United States, MayJune 2009. MMWR Morb Mortal Wkly Rep. 2009; 58: 1241-5.

21. Centers for Disease Control and Prevention. Antiviral agents for the treatment and chemoprophylaxis of influenza recommendations of the advisory committee on immunization practice (ACIP), United States: MMWR. 2010; 59: 1-28. 


\section{ANEXOS}

Cuadro 1. Comparación de las características clínico-epidemiológicas de los pacientes hospitalizados con infección respiratoria aguda grave con aquellos que fallecieron por la influenza pA(H1N1) en la delegación de Nuevo León del Instituto Mexicano del Seguro Social

\begin{tabular}{|c|c|c|c|}
\hline Variable & Casos $n=50$ & Controles $n=233$ & $\mathrm{p}$ \\
\hline \multicolumn{4}{|l|}{ Edad (años) } \\
\hline \multirow[t]{2}{*}{ Mediana } & 47 & 40 & 0.001 \\
\hline & & & Prueba de Mann-Whitney \\
\hline \multicolumn{4}{|l|}{ Tiempo de atención médica (días) } \\
\hline \multirow[t]{2}{*}{ Mediana } & 4 & 3 & 0.030 \\
\hline & & & Prueba de Mann-Whitney \\
\hline \multicolumn{4}{|l|}{ Género } \\
\hline Femenino & $25(50 \%)$ & $128(55 \%)$ & 0.520 \\
\hline Masculino & $25(50 \%)$ & $105(45 \%)$ & \\
\hline \multicolumn{4}{|l|}{ Embarazo } \\
\hline Mujer en edad fértil (15 a 49) & $16(100 \%)$ & $63(81 \%)$ & 0.060 \\
\hline Número de embarazadas & $0(0 \%)$ & $15(19 \%)$ & Prueba de Fisher \\
\hline \multicolumn{4}{|l|}{ Ocupación } \\
\hline Ama de casa & $11(22 \%)$ & $59(25 \%)$ & 0.810 \\
\hline Empleado & $17(34 \%)$ & $82(35 \%)$ & \\
\hline Otras & $22(44 \%)$ & $92(40 \%)$ & \\
\hline \multicolumn{4}{|l|}{ Lugar de residencia } \\
\hline Área Metropolitana & $48(96 \%)$ & $214(92 \%)$ & 0.300 \\
\hline Foráneos & $2(4 \%)$ & $19(8 \%)$ & \\
\hline \multicolumn{4}{|c|}{ Tratamiento con Inhibidores de neuroaminidasa } \\
\hline Sí & $8(16 \%)$ & $36(15 \%)$ & 0.920 \\
\hline No & $42(84 \%)$ & $197(85 \%)$ & \\
\hline \multicolumn{4}{|c|}{ Antecedente vacunal con vacuna antiinfluenza } \\
\hline Sí & $3(6 \%)$ & $14(6 \%)$ & 1.000 \\
\hline No & $219(94 \%)$ & $47(94 \%)$ & \\
\hline \multicolumn{4}{|l|}{ Comorbilidad y/o factor de riesgo } \\
\hline \multicolumn{4}{|l|}{ EPOC } \\
\hline Sí & $0(0 \%)$ & $7(3 \%)$ & 0.610 \\
\hline No & $50(100 \%)$ & $226(97 \%)$ & Prueba de Fisher \\
\hline \multicolumn{4}{|l|}{ Asma } \\
\hline Sí & $1(2 \%)$ & $10(4 \%)$ & 0.690 \\
\hline No & $49(98 \%)$ & $223(96 \%)$ & Prueba de Fisher \\
\hline \multicolumn{4}{|l|}{ Inmunodeficiencia } \\
\hline Sí & $3(6 \%)$ & $15(6 \%)$ & 1.000 \\
\hline No & $47(94 \%)$ & $218(94 \%)$ & Prueba de Fisher \\
\hline \multicolumn{4}{|l|}{ Diabetes } \\
\hline Sí & $11(22 \%)$ & $26(11 \%)$ & 0.040 \\
\hline No & $39(78 \%)$ & $207(89 \%)$ & \\
\hline \multicolumn{4}{|l|}{ Obesidad } \\
\hline Sí & $13(26 \%)$ & $18(8 \%)$ & 0.001 \\
\hline No & $37(74 \%)$ & $215(92 \%)$ & \\
\hline
\end{tabular}

Cuadro 2. Factores de riesgo de muerte de la cohorte de pacientes hospitalizados con infección respiratoria aguda grave debido a influenza pA(H1N1)

\begin{tabular}{lccc}
\hline Variable & Odds ratio crudo & Intervalo de Confianza 95\% & p \\
\hline EPOC & - & - & 0.210 \\
Diabetes & 0.240 & $1,02-4,95$ & 0.040 \\
Asma & 0.460 & $0,05-3,65$ & 0.450 \\
Inmunodeficiencia & 0.920 & $0,26-3,34$ & 0.910 \\
Obesidad & 4.190 & $1,85-9,49$ & 0.000 \\
Embarazo* & - & - & 0.060 \\
Tratamiento con inhibidores de neuroaminidasa & 1.040 & 2.400 & 0.920 \\
Antecedente vacunal con vacuna antinfluenza & 0.990 & $0,27-3,62$ & 1.000 \\
\hline
\end{tabular}

*Mujeres en edad fértil 15 a 49. 
Cuadro 3. Factores de riesgo de muerte de la cohorte de pacientes hospitalizados con infección respiratoria aguda grave debido a influenza pA(H1N1). Análisis multivariado

\begin{tabular}{lccc}
\hline & Odds ratio ajustado & Intervalo de Confianza 95\% & $\mathrm{p}$ \\
\hline Edad & 1.030 & $1,01-1,05$ & 0.001 \\
Tiempo de atención médica & 1.030 & $1,00-1,07$ & 0.090 \\
Asma & 0.620 & $0,07-532$ & 0.650 \\
Inmunodeficiencia & 0.980 & $0,25-3,79$ & 0.980 \\
Diabetes & 1.170 & $0,46-2,94$ & 0.730 \\
Obesidad & 4.440 & $1,85-10,6$ & 0.001 \\
\hline
\end{tabular}

* Odds ratio ajustado por edad, tiempo de atención médica, diabetes, asma, inmunodeficiencia y obesidad. 